\title{
Studies of intrinsic resolution to low energy electron and muon neutrino events with neutrino telescopes
}

\author{
Jannik Hofestädt* and Clancy W. James \\ for the KM3NeT Collaboration \\ Friedrich-Alexander University of Erlangen-Nürnberg, \\ Erlangen Centre for Astroparticle Physics \\ E-mail: Jannik.Hofestaedt@physik.uni-erlangen.de, \\ Clancy. James@physik.uni-erlangen.de
}

Existing large-volume neutrino telescopes such as ANTARES and IceCube, as well as the future KM3NeT/ARCA, investigate neutrinos at characteristic particle energies of $10 \mathrm{TeV}$, whereas KM3NeT/ORCA and PINGU will operate around $10 \mathrm{GeV}$ to determine the neutrino mass hierarchy by measuring the energy and direction of atmospheric neutrinos. In this energy regime, intrinsic fluctuations in particle behaviour become important.

These intrinsic fluctuations are investigated to answer two basic questions. Firstly: How do intrinsic fluctuations limit the reconstruction accuracy of an perfect detector, i.e. if every single photon is detected? While this requires making some basic assumptions about the methods used in the reconstruction, the answer to this question will indicate the optimum that could be achieved by any detector. Secondly: Given that only a finite number of photons will be detected, what is the best possible reconstruction accuracy in the case of a ideal use of the information carried by these photons? For this study the baseline design of the KM3NeT/ORCA detector is used.

This investigation separately considers muon tracks, and electromagnetic and hadronic cascades. By combining these results limits on the best possible reconstruction accuracy in energy and direction for the initial neutrinos achievable with an ORCA-type detector are derived.

The 34th International Cosmic Ray Conference,

30 July- 6 August, 2015

The Hague, The Netherlands

\footnotetext{
* Speaker.
} 


\section{Introduction}

It has recently been suggested that the neutrino mass hierarchy can be determined with multimegaton Cherenkov detectors in water or ice by measuring atmospheric neutrinos in the energy regime of $\sim 1-20 \mathrm{GeV}$ that have passed through the Earth towards the detector [1]. In order to resolve the hierarchy, the detector has to determine the neutrino arrival direction and energy, as well as identify the neutrino-interaction type.

Two detectors have been proposed to perform this measurement: ORCA [2], as part of KM3NeT, the future deep-sea neutrino telescope in the Mediterranean Sea; and PINGU [3], as a infill array of the IceCube detector at the South Pole. Both detectors would consist of large 3-dimensional arrays of photomultiplier tubes (PMTs) in water/ice that detect Cherenkov light emitted by charged particles emerging from neutrino reactions. The properties of the incoming neutrino can be inferred from the timing and amplitude of recorded PMT signals. The technology and design is similar to that of the existing neutrino telescopes ANTARES and IceCube, which search for astrophysical neutrinos at characteristic energies of $1 \mathrm{TeV}$ and above, although with a denser PMT array to compensate for the lower neutrino energies.

Both collaborations have produced preliminary estimates of the capability to resolve the neutrino properties with the proposed ORCA and PINGU detectors. The realism of such estimates should be evaluated against possible future improvements in the analysis techniques and detector layout modifications. However, several physical effects will limit the performance of such detectors, no matter how sophisticated the reconstruction methods or how dense the detectors are.

In this work, the limits imposed by intrinsic fluctuations in particle behaviour at energies of $1-20 \mathrm{GeV}$ on the energy and direction resolution of muon tracks, electromagnetic cascades and in particular hadronic cascades for an ORCA-like detector are derived by means of analysing the output of simulations. By combining these results and taking the kinematics of the neutrino interactions into account, the intrinsic limitations on the initial neutrino energy and direction resolution are derived.

\section{Method}

Neutrino telescopes detectors determine the properties of neutrino interaction events by measuring photons. For charged-current (CC) interactions, information from both the outgoing lepton (either a muon or electron - tau events are not considered here) and the hadronic cascade can be combined to reconstruct the primary neutrino energy and direction, while for neutral-current (NC) interactions, the outgoing neutrino is invisible to the detector. Ideally, photons from each of these components - muon tracks, electron cascades and hadronic cascades - could be uniquely identified, so that the capability to reconstruct neutrino events would only be limited by the resolution of each component when considered in isolation. This idealisation is used throughout. Indeed, optimistic assumptions are made at all stages, so that the performance of a real detector will always be worse than the resulting intrinsic limits. Always the 'forward' problem is considered, i.e. fluctuations in the observables are studied for a given simulated truth, rather than maximising the likelihood for given observables. Intrinsic limitations to energy and direction resolution of the three components are studied for two different idealized detectors: 'perfect' and 'finite' detector. 
Perfect detector is a infinitely large detector (every event contained in the detector volume) with a infinitely large PMT density, so that every single emitted photon is detected. Perfect timing and position calibration is assumed. Optical background and atmospheric muon background are not considered. Information carried by each photon is assumed to be perfectly used in event reconstruction. However, the same kind of reconstruction principles as for the finite detector are applied.

Finite detector is defined similarly to the 'perfect' detector, but with a finite PMT density, so that only a small number of photons are detected. Thus, the reconstruction accuracy will also be limited by the information carried by these detected photons. For this study, the characteristics of the proposed ORCA detector ${ }^{1}$ [2] are used to determine the photon detection probability (wavelength dependent, up to $\sim 3 \times 10^{-4}$ per photon @ $440 \mathrm{~nm}$ ). The effect of 'clumpiness' of the detector is not taken into account. Clumpiness is the fact that photon collection area is concentrated in small regions, i.e. PMTs, which in turn might be grouped in optical modules, and results in strong correlations between the photons that are detected.

For the finite detector, we optimistically assume that unscattered photons can be identified perfectly. Unscattered photons are in general used in direction reconstructions, as these photons carry directional information of the particle that emitted them.

\subsection{Muon tracks}

At the considered energies muons behave as minimum ionising particles, i.e. they undergo a constant energy loss per unit track length and travel on approximately straight lines. Therefore, the muon energy can be reconstructed by two obvious methods: the total light yield and track length.

Muon energy via total light yield is measured by simply counting the total number of detected photons (scattered and unscattered), which is proportional to the primary muon energy.

In the case of a perfect detector, relative fluctuations in the total number of emitted photons $N$ correspond directly to the relative energy reconstruction error:

$$
\Delta E_{\mu, N} / E_{\mu}=\sigma_{N} / N
$$

In the finite detector case, on average only $N^{d}$ photons are detected, which adds a extra Poissonian variation:

$$
\Delta E_{\mu, N}^{d} / E_{\mu}=\sqrt{N^{d}} / N^{d} .
$$

Muon energy via muon range is measured by the distance from the start point to the endpoint of a muon track. The track length increases nearly linear with the primary muon energy. In general, the emission positions of unscattered photons are estimated by back-projecting the detected photons onto the muon track assuming the Cherenkov hypothesis. The best possible resolution is achieved by assuming that the true emission positions of unscattered photons are detected directly.

In case of perfect detector, relative fluctuations in the muon range $L$ correspond directly to the relative energy reconstruction error:

$$
\Delta E_{\mu, L} / E_{\mu}=\sigma_{L} / L
$$

\footnotetext{
${ }^{1}$ The ORCA detector geometry are currently subject to optimisation. The average photocathode area density of the current detector configuration [2] with a vertical spacing of $6 \mathrm{~m}$ is assumed.
} 
In the finite detector case, the small number of detected unscattered photons results in an additional constant uncertainty $\Delta L^{d}$ from the detection of the muon endpoint.

Additionally, we assume that the muon track starting point, which is always the neutrino interaction vertex, can be resolved perfectly using information from the accompanying hadronic cascade, so that the reconstruction is reduced to a muon endpoint measurement.

Muon direction is in general reconstructed by a straight line fit to the muon track using the timing of detected photons. For a perfect timing, the intrinsic limitation of this procedure is the straightness of the full muon track itself. Therefore, we estimate the intrinsic limits on the muon direction from straight line fits to the true muon tracks.

However, it is difficult to devise a suitable limit on the direction resolution in the case of a finite detector, since only a few photons are needed to constrain the reconstruction. Thus, we assume the finite detector limit to be the same as that for a perfect detector.

\subsection{Cascades}

An energetic electron initiates a purely electromagnetic cascade, while the W/Z-boson produced in CC/NC interactions of (anti)neutrinos with nucleons initiates a hadronic cascade $h$, although in the few-GeV regime this hadronic cascade may consist of only a few particles. We define the energy and direction of such a hadronic cascade $\mathbf{p}_{h}$ through four-momentum conservation at the neutrino interaction vertex: $\mathbf{p}_{h}\left(E_{h}, \vec{p}_{h}\right) \equiv \mathbf{p}_{v}-\mathbf{p}_{\ell}$, where $\mathbf{p}_{v}$ and $\mathbf{p}_{\ell}$ are the four-momenta of the incoming neutrino and outgoing lepton, respectively. This definition is chosen, because it is the most relevant for the reconstruction of the incoming neutrino.

We assume that electromagnetic and hadronic cascades are reconstructed using the same methods.

Cascade energy is estimated by simply counting all detected photons. For electromagnetic cascades the number of emitted photons is proportional to the cascade energy, while for hadronic cascades the light yield increases faster than linear with the cascade energy and approaches asymptotically the light yield of electromagnetic cascades ${ }^{2}$. This behaviour is taken into account in the energy estimated for hadronic cascades. Fluctuations for the perfect and finite detector case are similar to that of the muon energy estimation via the total light yield, cf. Sec. 2.1.

Cascade direction is in general reconstructed by fitting the angular emission profile around trial cascade axes (for a given vertex position). We estimate the error of such reconstruction methods by the fluctuation in the mean direction of detected unscattered photons.

If $\hat{v}$ is the normalised mean photon direction and $\hat{c}$ is the normalised cascade direction, the angular error is given by:

$$
\Delta \theta=\cos ^{-1}(\hat{v} \cdot \hat{c}) .
$$

The resulting distributions from many simulated electrons are well described by two-dimensional Gaussians (with a rotational symmetry around the cascade axis) on a sphere. For sufficiently high energies this approximation holds also for hadronic cascades.

\footnotetext{
${ }^{2}$ In each secondary interaction a substantial fraction of produced particles are $\pi^{0}$, which increase the electromagnetic activity within hadronic cascades. With increasing cascade energy, the cascade becomes more electromagnetic-like.
} 
A very important difference between the intrinsic limits in the case of electron cascades compared to hadronic cascades is that the initial electron is always identical, while hadronic cascades with the same energy $E_{h}$ may consist of different sets of particles produced at the vertex. This effect is in addition to the effects of random particle propagation, which causes fluctuations for the same initial set of particles. Therefore, in the case of hadronic cascades we separate the resolution estimates into two components: that due the choice of initial particles at the 'vertex' and that due to random particle 'propagation'. In this nomenclature, cascades initiated by single electrons show only propagation variations but no vertex variations.

The two components can be measured individually by simulating many different vertices and each vertex many times: the variation due to propagation is given by the variation of individual iterations of a given vertex compared to the mean properties over all iterations of that vertex, while the vertex variation is given by the variation of the mean properties between different vertices.

\subsection{Simulations}

Particle propagation, and Cherenkov light emission and propagation in seawater is simulated using a standard simulation package of the ANTARES collaboration [4], which is a GEANT 3.21 implementation. Hadron tracking is performed by GEISHA. A comparison with the version of FLUKA in GEANT shows relative differences in the mean Cherenkov light yield and its fluctuations due to different particle propagations of about $10 \%$ for hadronic cascades with $E_{h}=10 \mathrm{GeV}$.

Simulations are performed at discrete energies between $1-20 \mathrm{GeV}$, with hadronic cascades extracted from $v_{\mu}$ CC induced interactions ${ }^{3}$ with seawater generated with a software package based on the widely used GENIE [5].

\section{Results}

For the energy resolution we use the RMS as a measure of the fluctuations, while for the direction resolution the $68.27 \%$ quantile (equivalent to \pm 1 Gaussian $\sigma$ ) is used.

\subsection{Muon tracks}

The relative muon energy resolution for both considered energy estimates is shown in Fig. 1 (right). Using the number of detected photons, our simulations indicate that 1.15 detected unscattered photons from the last $73 \mathrm{~cm}$ of the muon track are expected, corresponding to $\pm 1 \sigma$ for Poissonian photon emission. This results to roughly $\sim 0.1 \mathrm{GeV}$ uncertainty on the muon energy. While the relative muon energy resolution from the total light yield method scales with $\sqrt{E}$ for the finite detector, relative fluctuations in the true muon range slightly increase with energy due to rare significant energy losses along the track. For muon energies below $E_{\mu} \lesssim 12 \mathrm{GeV}$ the limitation on energy estimation via the muon range method is better than $6.5 \%$ and below that of the total light yield method.

Limitations on the muon direction due to deviations of the true muon track from a straight line are roughly $0.7^{\circ}-2^{\circ}$ in the considered energy range. This is negligible compared to the resolutions from hadronic cascades, see Sec. 3.2.

\footnotetext{
${ }^{3} \mathrm{~A}$ preliminary study indicated that hadronic cascades from $v / \bar{v} \mathrm{CC}$ and $\mathrm{NC}$ are similar, in line with expectations.
} 

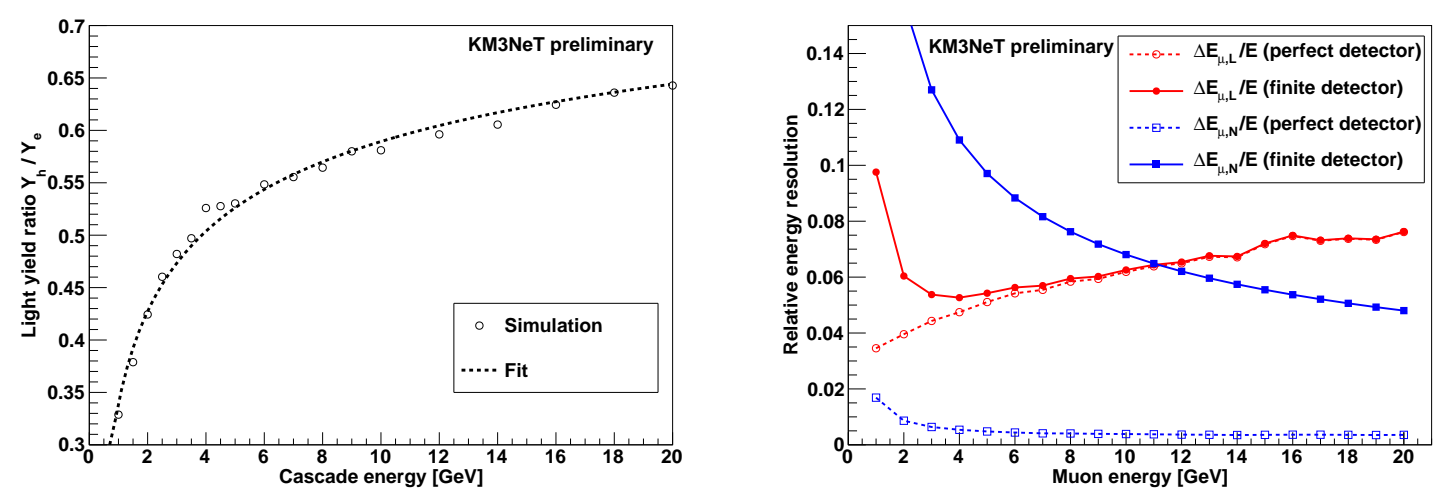

Figure 1: Left: ratio $F$ of the mean number of emitted photons from hadronic and electromagnetic cascades as a function of cascade energy $E$. A fit is shown as dashed line. Right: limits on the relative muon energy resolution as a function of muon energy for the muon range method $\Delta E_{\mu, L}$ (red) and the total light yield method $\Delta E_{\mu, N}$ (blue).

\subsection{Cascades}

The mean number of detected photons from cascades initiated by an electron in the finite detector case is $N=20.8 \times E_{e} / \mathrm{GeV}$. From these photons $50.4 \%$ are unscattered. Fluctuations in the number of emitted photons are below $1 \%$. This is the limiting resolution for a perfect detector. In case of a finite detector, the resolution is limited by the Poissonian $\sqrt{N}$ fluctuations.

The ratio of the mean number of photons from hadronic and electromagnetic cascades is shown in Fig. 1 (left) and is fitted similarly as in Ref. [6]. Variations in energy and direction estimates due to different sources of fluctuations (vertex, propagation and detection) for hadronic cascades with $E_{h}=5 \mathrm{GeV}$ are shown in Fig. 2. The energy resolution for a perfect detector (combination of both vertex and propagation variation) is approximately Gaussian for $E_{h} \gtrsim 5 \mathrm{GeV}$, while the direction resolution shows non-Gaussian tails which originate from the propagation variations. However, for the finite detector case the direction resolution becomes approximately Gaussian, as the contribution from the detection of only a small sample of photons dominates vertex and propagation variations.

The resulting limits for the energy and direction resolution are shown in Fig. 3 as a function of cascade energy. For hadronic cascades the energy resolution of a perfect detector is dominated by the vertex variations, while for the direction resolution the magnitude of vertex and propagation variations are compatible. Fluctuations due to detection of only a small photon sample dominates the direction resolution of a finite detector, but is subordinated for the energy resolution.

\subsection{Intrinsic resolutions of muon and electron neutrino $\mathrm{CC}$ events}

Using the results of the previous sections, intrinsic limitations on the neutrino properties can be deduced. We assume that the neutrino energy $E_{v}$ and normalised direction $\hat{p}_{v}$ are reconstructed by combining the estimated electron/muon properties $\left(E_{e, \mu}, \hat{p}_{e, \mu}\right)$ with that of the hadronic cascade $\left(E_{h}, \hat{p}_{h}\right)$ using energy and momentum conservation (neglecting electron/muon masses):

$$
E_{v}=E_{e, \mu}+E_{h} \quad \text { and } \quad \hat{p}_{v} \approx \frac{E_{e, \mu}}{E_{e, \mu}+E_{h}} \hat{p}_{e, \mu}+\frac{E_{h}}{E_{e, \mu}+E_{h}} \hat{p}_{h} .
$$



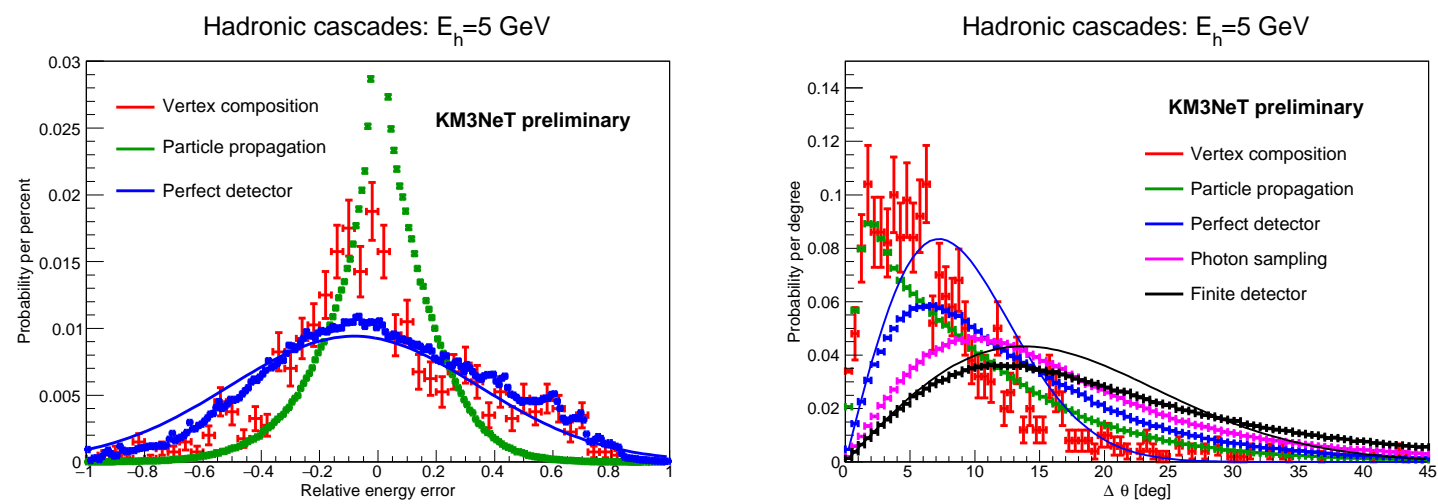

Figure 2: Distribution of relative energy error (left) and direction error (right) for hadronic cascades with $E_{h}=5 \mathrm{GeV}$, separated into different sources of fluctuations. Error bars represent Poissonian errors. For comparison, best-fit Gaussian approximations are shown as lines for the perfect and finite detector case.
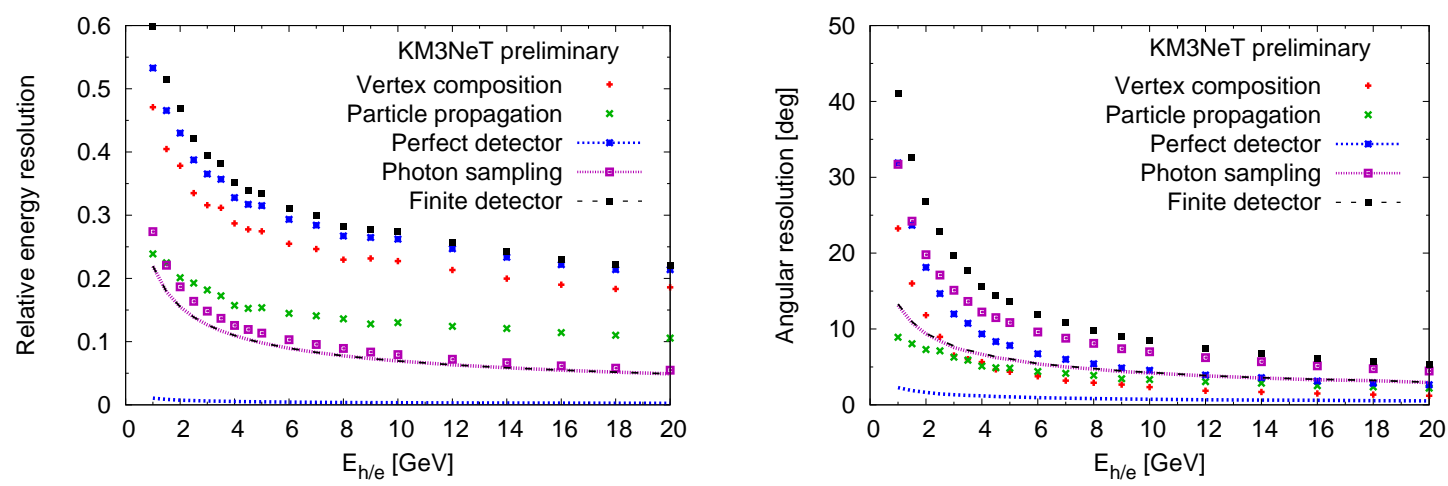

Figure 3: Relative energy (left) and direction (right) resolution of electromagnetic (lines) and hadronic cascades (markers) as a function of cascade energy, separated into different sources of fluctuations.

For the calculation of the neutrino direction resolution the correlations between energy and direction errors and the kinematics of neutrino interactions are taken into account. Fig. 4 shows the resulting limits on the neutrino energy and direction resolution for $v_{e}$ and $v_{\mu} \mathrm{CC}$ events as a function of neutrino energy and Bjorken $y, y \approx 1-E_{e, \mu} / E_{v}$, also known as inelasticity.

\section{Conclusions}

Intrinsic fluctuations in particle behaviour at energies of $1-20 \mathrm{GeV}$ impose stringent limits on the energy and direction reconstruction accuracy of muon tracks, electromagnetic cascades and in particular hadronic cascades for water/ice neutrino telescopes.

These limits assume 100\% neutrino event detection efficiency — implicit or explicit cuts on event quality can of course beat these limits, but only at the cost of inefficiencies.

The limitations for muon tracks and electromagnetic cascades are small compared to that of hadronic cascades. For hadronic cascades the fluctuations due to different vertices has the largest effect on the energy resolution, while for the direction resolution the contribution from detecting only a small sample of photons is relevant. 

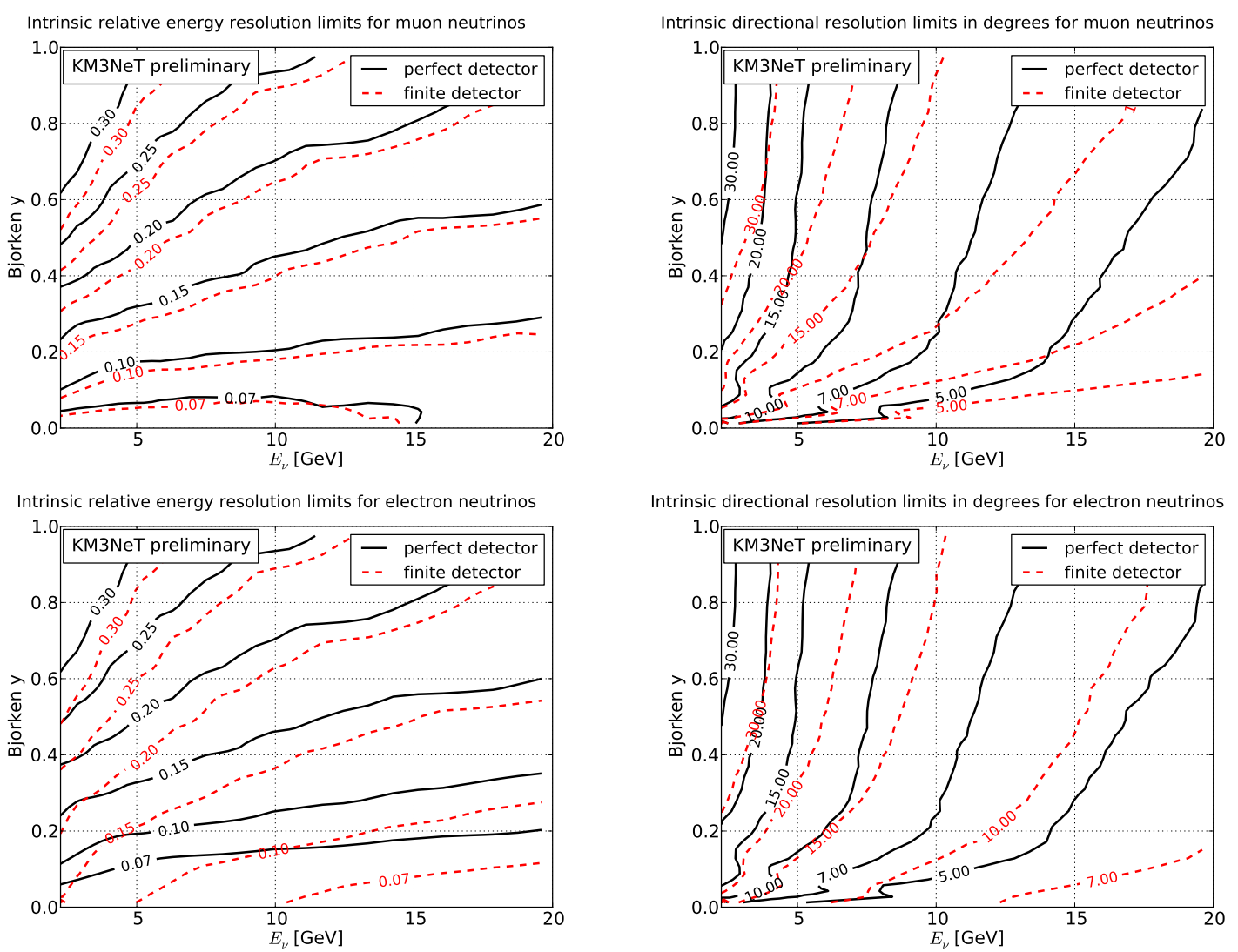

Figure 4: Limitations on the relative energy (left) and direction (right) resolution of neutrinos in $v_{e} \mathrm{CC}$ (top) and $v_{\mu}$ CC events (bottom) as a function of neutrino energy $E_{v}$ and Bjorken $y$. Resolutions for the perfect detector (solid black) and finite detector case (dashed red) are separately shown as contour lines.

For the assumed ORCA-like detector the neutrino energy resolution in $v_{e}$ and $v_{\mu}$ CC events is mainly affected by the irreducible fluctuations in the hadronic cascades itself. This indicates that under our optimistic assumptions a sparser detector with a larger volume might be better for the neutrino mass hierarchy measurement in water. However, this has to be studied in detailed simulations.

\section{References}

[1] E. Akhmedov, S. Razzaque, A. Smirnov, JHEP 1302 (2013) 082.

[2] J. Brunner, for the KM3NeT Coll., PoS(ICRC2015)1140.

[3] M.G. Aartsen et al., arXiv:1401.2046 [physics.ins-det] (2014).

[4] A. Margiotta, for the ANTARES Coll., Nucl. Instrum. Meth. A 725 (2013) 98-101.

[5] C. Andreopoulos et al., Nucl. Instrum. Meth. A 614 (2010) 87.

[6] M. Kowalski, Ph.D. thesis, Humboldt Universität Berlin (2004). 